\title{
Translating Therapies for Huntington's Disease from Genetic Animal Models to Clinical Trials
}

\author{
Steven M. Hersch* ${ }^{\dagger}$ and Robert J. Ferrante ${ }^{\ddagger \S}$ \\ *Neurology Service, Massachusetts General Hospital and Harvard Medical School, Boston, Massachusetts 02114; \\ ${ }^{\dagger}$ MassGeneral Institute for Neurodegenerative Disease, Massachusetts General Hospital, Charlestown, Massachusetts 02129; \\ ${ }^{\ddagger}$ Geriatric Research Education and Clinical Center, Bedford VA Medical Center, Bedford, Massachusetts 01730; and \\ ${ }^{\S}$ Neurology, Pathology, and Psychiatry Departments, Boston University School of Medicine, Boston, Massachusetts 02118
}

Summary: Genetic animal models of inherited neurological diseases provide an opportunity to test potential treatments and explore their promise for translation to humans experiencing these diseases. Therapeutic trials conducted in mouse models of Huntington's disease have identified a growing number of potential therapies that are candidates for clinical trials. Although it is very exciting to have these candidates, there has been increasing concern about the feasibility and desirability of taking all of the compounds that may work in mice and testing them in patients with HD. There is a need to begin to prioritize leads emerging from transgenic mouse studies; however, it is difficult to compare results between compounds and laboratories, and there are also many additional factors that can affect translation to humans. Among the important issues are what constitutes an informative genetic model, what principals should be followed in designing and conducting experiments using genetic animal models, how can results from different laboratories and in different models be compared, what body of evidence is desirable to fully inform clinical decision making, and what factors contribute to the equipoise in determining whether preclinical information about a therapy makes clinical study warranted. In the context of Huntington's disease, we will review the current state of genetic models and their successes in putting forward therapeutic leads, provide a guide to assessing studies in mouse models, and discuss some of the salient issues related to translation from mice to humans. Key Words: Huntington's disease, therapy, neuroprotection, neurodegenerative, transgenic mice, clinical trials.

\section{INTRODUCTION}

Genetic models have revolutionized the study of human neurological diseases by providing accurate and experimentally accessible systems in which to study molecular pathogenesis. Genetic models also provide an opportunity to test potential treatments and explore their promise for translation to humans experiencing these diseases. The promise of using genetic models for treatment discovery is perhaps greatest in inherited diseases, such as Huntington's disease (HD), which affect single genes. Although making the therapeutic leap from genetic models to humans fulfills one of the promises of molecular medicine, it also brings risk to human subjects

Address correspondence and reprint requests to Steven M. Hersch, M.D., Ph.D., MassGeneral Institute for Neurodegenerative Disease, MGH East Building 114-2001, 114 16th Street, Charlestown, MA 02129. E-mail: hersch@helix.mgh.harvard.edu; or Robert J. Ferrante, Ph.D., Geriatric Research Education Clinical Center Unit 182B, Bedford VA Medical Center, 200 Springs Road, Bedford, MA 01730. E-mail: rjferr@bu.edu. and expends human and financial resources. Thus, how data from experiments using genetic models are used to inform choices about clinical trials requires reflection and examination. Among the important issues are what constitutes an informative genetic model, what principals should be followed in designing experiments using genetic models, what body of evidence is desirable to fully inform clinical decision making, and what factors contribute to the equipoise in determining whether preclinical information about a therapy makes human study warranted.

Genetic models encompass a variety of assays in which the mutant gene or some portion of it has been introduced into living organisms which experience a phenotypic response in some measure indicative of the human disease. These include cell culture models in which the outcome measure is cytotoxicity or some structural or biochemical measure related to pathogenesis and which are especially suited for high-throughput screening, lower organisms such as Drosophila, Caenorhabditis elegans, or zebrafish, which provide a more 
salient context for the genetic mutation and opportunity for genetic analyses, and mammalian models, usually transgenic, knock-in, or knockout mice, in which the disease expression and response to potential therapies can be quite close to what occurs in humans. Candidate therapies may arise from experiments using any of these models based on hypotheses about the disease mechanisms. In addition, screening of high-throughput assays using libraries containing thousands to millions of small molecules can bring candidate therapies forward based on obtaining a desired biochemical or cellular phenotype, irrespective of knowledge about mechanism of action. However, as leads arise, proof of efficacy in mammalian models is considered a requisite before considering possible testing in humans. For compounds already in the pharmacopoeia, it can be easy to contemplate rapid translation to clinical trials. For compounds that are not yet pharmaceuticals or nutriceuticals, efficacy in mammalian models would lead first to standard preclinical pharmacokinetic, toxicity, teratagenicity studies, and perhaps the development of more promising analogs before testing can begin in humans. When selecting compounds for study in preclinical experiments, we have prioritized compounds already in human use because of the indispensable knowledge base that comes with them and the existence of pharmaceutical material ready for human use.

Transgenic mouse models of HD have been extensively used in the last several years to test potential therapies. Although most studies have concentrated on compounds that have already had human use, we are also beginning to test leads arising from screens performed in cellular and submammalian models. Some of the compounds that we have demonstrated to be efficacious in transgenic mice have gone on to clinical testing, often in trials we also participated in designing. Through this experience and discussions with many others, we have developed a perspective about using transgenic mouse experiments to stimulate and guide human clinical trials. We also encountered a wide range of thoughts in the HD research community, from the opinion that preclinical studies in mouse models are of limited significance because mice and humans are so different, to suggestions that there should be rigid criteria for success in the mouse models before a therapy is considered for clinical trials. It is quite complex to consider what constitutes sufficient data from mouse models to justify translation to humans. The lack of a proven neuroprotective therapy for HD also effects the equation by keeping the level of urgency high.

Because the numbers of clinical trials in HD have increased, as has the number of compounds with favorable transgenic mouse data, there has been increasing concern about the feasibility and desirability of taking all the existing drug compounds that may work in mice and testing them in HD patients. Though there is a need to begin to prioritize leads emerging from transgenic mouse studies, it may be difficult to compare results between compounds and laboratories. There are also many additional factors that can affect the overall desirability of compounds, such as availability from a manufacturer, expected toxicity, redundancy of mechanism, and the nature of potential interactions with other agents. As yet, there have been insufficient fully powered clinical trials in humans to permit a comparison of therapeutic benefits in the two species (mice and human) for any given therapy. Thus it has not yet been confirmed that experiments demonstrating improved phenotype in transgenic mice are predictive of benefits in humans. Similarly, it is unknown whether the magnitude of benefit in mice predicts the magnitude of benefit in humans. Nevertheless, results from human clinical trials will illuminate the value of mouse clinical trials in the foreseeable future. In the meantime, there are clinical trials being planned, a growing list of therapies that are effective in mouse models, and a need for discussion about how transgenic mouse therapeutic trial data can best be used. In the context of Huntington's disease, we will review the current state of such studies, provide a guide to assessing them, and discuss some of the salient issues related to translation from mice to humans.

\section{Genetic mouse models of HD}

The ideal transgenic mouse model would have the following: 1) a robust phenotype, 2) rapid disease onset and progression, 3 ) well defined neurobehavioral abnormalities, which can be quantified, 4) neuropathological findings which accurately mirror human HD, and 5) limited variability to enhance study power. Multiple mouse models of HD now exist, falling into three broad categories: 1) mice expressing fragments, usually the first one or two exons of the human huntingtin gene that contain the polyglutamine expansion (in addition to both alleles of murine wild-type huntingtin, $H d h$ ), 2) transgenic mice expressing the full-length human $H D$ gene with an expanded polyglutamine tract (plus the murine $H d h$ ), and 3) knock-in mice with pathogenic CAG repeats inserted into the existing CAG expansion in murine $H d h$. All of these models share features with human HD. Not surprisingly, the degree of similarity to human HD increases the closer the model reproduces the exact neuropathological and molecular conditions for HD. Unfortunately, the more genetically accurate the model, the more variable and subtle the phenotype. Thus, it has so far been much more feasible to use the fragment models for therapeutics research because the outcomes are more prevalent and definable; hence, trials can be conducted using smaller sample sizes. An emerging strategy is to use the fragment models for most experiments and fulllength transgenic or knock-in mice for confirmatory studies once the potential of a compound has been established. Until there is more feedback from human ther- 
apeutic trials, it will not be known whether the fragment mice can stand on their own as predictors of responses to therapies or whether the full-length models are even necessary. Meanwhile, efforts continue to optimize phenotypes for practical use as therapeutic outcomes. We will describe some of the mouse models to illustrate how they can be used and some of their differences.

The first transgenic mouse model of HD was the R6/2 mouse which has a small $\mathrm{N}$-terminal fragment (exon 1) of huntingtin with $\sim 150 \mathrm{CAG}$ repeats. ${ }^{1}$ The R6/2 model has a well characterized progressive phenotype with moderate variability such that experimental groups can contain as few as 10 mice and provide the power to detect $10 \%$ differences in many outcome measures. It is possible to perform survival studies, an important potential surrogate indicator for neuroprotection, in approximately 3 months. R6/2 mice have been used in a number of preclinical therapeutic trials. Although the R6/2 model displays many of the behavioral and neuropathological features observed in HD patients, it is not a perfect genetic and neuropathological match to what is observed in humans. In particular, the R6/2 mice have more extensive huntingtin aggregate formation than occurs in $\mathrm{HD}$, they are resistant to excitotoxicity, and neuronal loss is less selective and dramatic. Nevertheless, the efficiency and clear experimental endpoints of the R6/2 mice remain a major advantage; ${ }^{2}$ thus, they have been used extensively.

The N-171-82Q mice have a longer N-terminal fragment of huntingtin (exon 1 and 2) with 82 polyglutamines. ${ }^{3}$ These mice have a less well defined neurobehavioral phenotype than the R6/2 mice. Their neuropathological features are more similar to human HD in that huntingtin aggregates are more prominent in cortex than in striatum and neurodegeneration is more prominent and seems more selective for the striatum. ${ }^{4}$ However, the phenotype is more variable than that observed in the R6/2 mice, and therefore a much larger number of mice are necessary to provide adequate power; a minimum of 20 mice in each treatment group is necessary for many outcome measures used in preclinical drug trials. We and others found the N171-82Q mice to be a useful model for testing potential therapies. ${ }^{5-10}$ Interestingly, dietary restriction of these mice improves survival by about 2 weeks. ${ }^{11}$

Transgenic mice with full-length huntingtin containing 48 or 89 CAG repeats have been described that show behavioral abnormalities and neuron $\operatorname{loss}^{12}$ but have not been available for use in therapeutic discovery. A yeast artificial chromosome mouse model of HD with the entire human huntingtin gene containing $128 \mathrm{CAG}$ repeats (YAC 128), as well as flanking segments that might include regulatory elements, develops motor abnormalities consisting of initial hyperactivity followed by difficulty walking along an elevated rotating rod from 6 to 12 months, and then hypokinesis. ${ }^{13}$ These mice show a loss of cortical and stri- atal neurons, which correlate with behavioral changes. Although neuropathology has greater fidelity with human HD, variability and the length of time for phenotype development may hinder the use of this model for treatment paradigms. New transgenic mice, created using bacterial artificial chromosomes, look promising. ${ }^{14}$ These mice have 226 CAG repeats in the full-length human huntingtin gene. They show movement abnormalities such as tremor, head bobbing, circling, and hyperactivity around 10 weeks of age. At 4-5 months they become hypoactive and, subsequently, die at 6-7 months of age. They have selective neuronal loss in both the striatum and cerebral cortex. Although transgenic mice expressing a full-length mutant huntingtin have not yet been used in a published therapeutic discovery experiment, there are many efforts underway.

Knock-in mice represent the most precise genetic model of HD and a number of lines have been made. A knock-in Hdh mouse with 72-80 CAG repeats shows aggressive behavior and neuropil aggregates, but no gliosis or neuronal loss. ${ }^{15,16}$ The $H d h^{Q I I I}$ knock-in mice have $111 \mathrm{CAG}$ repeats inserted into the murine HD gene. ${ }^{17}$ The mice develop a progressive neuropathological phenotype with specificity for striatal neurons, consisting of nuclear localization of the full-length huntingtin protein in medium spiny neurons, and subsequent formation of N-terminal inclusions and insoluble aggregates. The mice eventually develop a mild depletion of striatal neurons, although they do not develop a behavioral phenotype. ${ }^{18}$ Another knock-in mouse model with 150 CAG repeats has late-onset gait abnormalities and develops neuronal intranuclear inclusions predominantly in the striatum. ${ }^{15}$ Lastly, a knock-in mouse with $94 \mathrm{CAG}$ repeats has increased rearing at night at 2 months of age, decreased activity at 4 and 6 months, but a normal lifespan. ${ }^{19}$ Nuclear microaggregates were present at 4 months and were widely distributed at 6 months of age in these mice. So far, the knock-in mouse lines do not have a sufficient expression of disease to use progressive morbidity and survival as an endpoint; however, they do have a variety of measurable neuropathological and behavioral phenotypes that could be validated as potential endpoints in therapeutic studies.

\section{Methodological considerations for mouse therapeutic trials}

As in human clinical trials, the use of appropriate methodology in mouse trials determines whether the experimental outcomes are valid and useful. Many of the same considerations apply to both human and mouse trials but are not always considered in the latter. For example, blinding of study personnel about the treatment condition of the animals they are studying should be standard practice. Power analyses are necessary to establish whether the study has sufficient numbers of mice for each endpoint. Whereas in human trials enormous vari- 
ability in clinical measures is common, in mouse trials it is possible to minimize measurement variability and thereby increase the power to detect smaller differences in smaller groups. It is important to understand the genetic, physical, and environmental sources of variability to take advantage of this.

An estimation of the sample size is a critical first step. The numbers of mice for preclinical studies must be carefully considered and reflect the different outcome measures to be analyzed. Our experience with survival studies has been that a minimum sample size of 10 per treatment group in the R6/2 transgenic mice and a sample size of 20 per group in the N171-82Q transgenic mice are necessary to have an $80 \%$ power of detecting a $30 \%$ difference between groups. Our experience with neurohistopathological measures and neurochemical assays has shown that a standard deviation of $15-20 \%$ can be expected. A similar power analysis suggests that to detect a $30 \%$ difference between groups a sample size of 10 is necessary for each cohort.

As in human clinical research, it is important to have inclusion and exclusion criteria, which should be predetermined. For example, there is good reason to consider excluding "runts" (we routinely exclude mice weighing less than $8 \mathrm{~g}$ at 21 days), mice with injuries, mice in which there is drift of their transgene (which must be monitored), and mice unable to perform some of the planned evaluations, such as the rotarod. ${ }^{2}$ Treatment groups should be genetically comparable. In particular, there should be methods to prevent overrepresentation of sibs in any group. For example, it can be predetermined that no more than one or two mice from any litter be in a given experimental group. The environment in which these mice are housed and treated needs to be uniform, because environmental enrichment slows disease progression. ${ }^{20}$ Another important consideration is the onset and duration of treatment in mouse trials. Initiation of treatment at weaning, as has been most common, is analogous to treating presymptomatic humans. Initiation of treatment once a motor or other phenotype is evident would be analogous to most human trials for HD, which have generally used early symptomatic subjects. There has only been one mouse trial published, which tested creatine, in which treatment was initiated during symptomatic stages. ${ }^{21}$

There are many potential outcome measures that can be used in mouse therapeutic trials. These bear close examination because they can differ enormously in relevance with some clearly being much more informative and specific than others. In neurodegenerative disease research we are especially concerned with achieving neuroprotection, which at its most basic level is the preservation of neuronal processes, somata, and function. Measures that assess these directly (brain weight, gross atrophy, cellular atrophy, neuronal counts, gliosis, volu- metric imaging) should be considered the primary outcome measures. Although it may also be possible to model the treatment of symptoms in genetic models and it may also happen that improving symptoms corresponds to neuroprotection, assessments of symptoms should be considered secondary outcome measures. The reason for this is that symptoms can be modified without affecting neurodegeneration; for example, by modifying motivation, level of consciousness, energy, etc. This distinction is important to keep in mind when considering how informative the results of a mouse therapeutic trial might be for considering translation to humans. A treatment cannot be considered to be neuroprotective in mice in the absence of neuropathological evidence that brain atrophy, cellular atrophy, or neuronal loss has been prevented. The quality of neuropathology in mouse therapeutic trials is therefore of foremost importance. It should also be mentioned that other neuropathological measures, such as protein aggregate load or the expression of molecules of interest, are only meaningful if brain atrophy, cellular atrophy, or neuronal loss are measured to provide a context for interpreting them.

In our labs, cohorts of treated and untreated wild-type and mutant littermate mice are euthanized at early, moderate, and late stages of their phenotypes for neuropathological analysis. Standardization of tissue processing is critical to the analysis. As an example, cut tissue sections mounted from water will expand and those mounted from phosphate buffer will contract, resulting in entirely different brain measures. Brain specimens are identified by the originally assigned code so that the subsequent studies are performed blind to the investigator as to the genetic identity and treatment of the mice. Quantitative methods are essential, because observation alone can only detect large differences and semiquantitative methods are prone to many types of errors. We use stereology to obtain various brain and cellular volumes and counts and also to quantify other pathological features, such as huntingtin aggregates.

Secondary outcome measures include survival, body weight, performance on motor and cognitive tasks, and laboratory studies examining toxicity or putative mechanisms of action. Mouse survival or a surrogate for mouse survival, which are usually criteria for advanced morbidity that agrees with the animal care policies of an institution and triggers euthanasia, is an especially useful secondary outcome measure. Survival provides a useful first-pass screen that can be assessed as the main outcome in pilot studies, analogous to phase II studies in humans, to assist in deciding whether more extensive studies are warranted and to help design them. Besides generally correlating well with neuropathology, it provides a relevant measure of the magnitude of benefit that is both understandable and enables ready comparison with other therapies. A dose versus survival study is 
recommended, using at least three doses, to help determine an optimal dose and to learn whether therapeutic benefits stabilize (saturate) or decline past that optimum. It is important to observe mice in any treatment paradigm twice daily throughout the experiment for any signs of morbidity and mortality. Strict criteria are necessary to determine when mice should be euthanized. Although this may vary between institutions, our own criteria for euthanization is the point in time when the mice are unable to initiate movement and right themselves after being placed upon their side and gently prodded for $30 \mathrm{~s}$. Two independent observers confirm these criteria.

Although body weight has often shown early and significant improvements in some mouse therapeutic trials, little effect has also been observed in other trials despite dramatic improvement in survival. Our practice is to measure body weight weekly, on the same day, usually from the time of weaning. Diurnal feeding habits necessitate that weights be taken at the same time, morning or afternoon. As mice progress toward the latter stages of disease, we usually increase the frequency of weighing to twice weekly.

A variety of motor tasks have been used in mice to assess the clinical phenotype. These include measuring activity and grooming, the length of time a mouse can balance on a rotating rod, grip strength measurement, and gait. Preserved motor performance closely correlates with improved survival and neuroprotective outcomes, though compounds affecting the CNS can also have pharmacological effects. The rotarod test, in which mice are required to walk along an elevated rotating rod, is generally accepted as the most sensitive and encompassing and is widely used. This equipment is commercially available and its use has been standardized within most laboratories. In the fragment models, we perform testing twice weekly. There are a number of variations of rotational speed and whether the rod is at a constant speed or accelerating, which investigators have applied. The length of time that the mice remain on the rotating rod is used as the measure of competency on this task. Mice are tested until they are unable to perform. On any given day, mice may fail or refuse to perform this task. If this occurs early on in the time course of disease phenotype, additional repeated trials the next day may be useful to ensure that performance failure is a reflection of disease. In the absence of a rotarod apparatus, the onset of forelimb and hindlimb clasping, a measure of neurological abnormality in HD mice, can be assessed daily until identified and confirmed by a second investigator. Interrater variability and methodological differences between labs can make it difficult to use motor tasks for comparing the relativity efficacy of one treatment to another.

Declining cognition occurs early in HD and the mouse models are expected to develop cognitive deficits as well. However, few cognitive assessments have been validated in the mouse models currently in use. In the fragment models, it is difficult to complete training before the phenotype has already compromised performance. Nevertheless, water maze and prepulse inhibition have been used by a number of investigators and efforts to develop further cognitive tests, such as serial reaction time and other implicit memory tasks are ongoing. Cognitive endpoints may prove valuable in the full-length models in which motor symptoms are slow to develop.

Open Field Testing, which assesses general activity, is a complex measure that may be most useful in the fulllength models that progress more slowly. Commercially available units to detect movement can analyze and quantify motor behaviors. Testing is usually performed during the dark phase of the diurnal cycle when mice are most active. Measurements can be made during the first 15-30 min interval after placement in a novel open field. Horizontal and vertical motions can be counted and locomotor activity, rearing, circling, and grooming assessed. The open field box must be cleaned between mice to prevent behavioral effects from the odors of previous occupants. Testing is performed once a month to avoid habituation to the open field and to ensure novelty of the measured behavior. There can be some specific differences in the patterns of behaviors observed. For example, some HD mice seem to continually repeat an abortive grooming process.

A behavioral observation system, SHIRPA, is a threestage semiquantitative protocol designed to characterize phenotype changes in mice. In therapeutic trials, behavioral and functional changes are scored by an observer blinded to treatment. ${ }^{22} \mathrm{~A}$ composite score is calculated following the assessment. A modified SHIRPA has been developed for longitudinal use in mice with neurological disease. ${ }^{23}$ The utility of such a behavioral protocol is in its standardization and may be a useful cross-measure for all laboratories performing therapeutic drug trials in mice with neurological disease. The scoring, however, requires detailed scrutiny of some behaviors and function in HD mice that are subject to interpretation, such as limb clasping and physical appearance. For this reason, it is important to maintain observer consistency throughout a study.

Several types of laboratory studies contribute crucial information that can greatly enrich the preclinical information about a compound. Pharmacokinetic analysis of the study compound, its metabolites, or of molecules expected to be affected by the treatment help insure that the compound reaches the brain and performs as expected. Pharmacokinetic studies are useful in combination drug trials to examine the effects on bioavailability of one compound on the other compound. Additionally, understanding effective doses and brain levels in mice can set the stage for asking whether such doses and levels can be achieved in humans. In our experience, 0.5-1.0 ml 


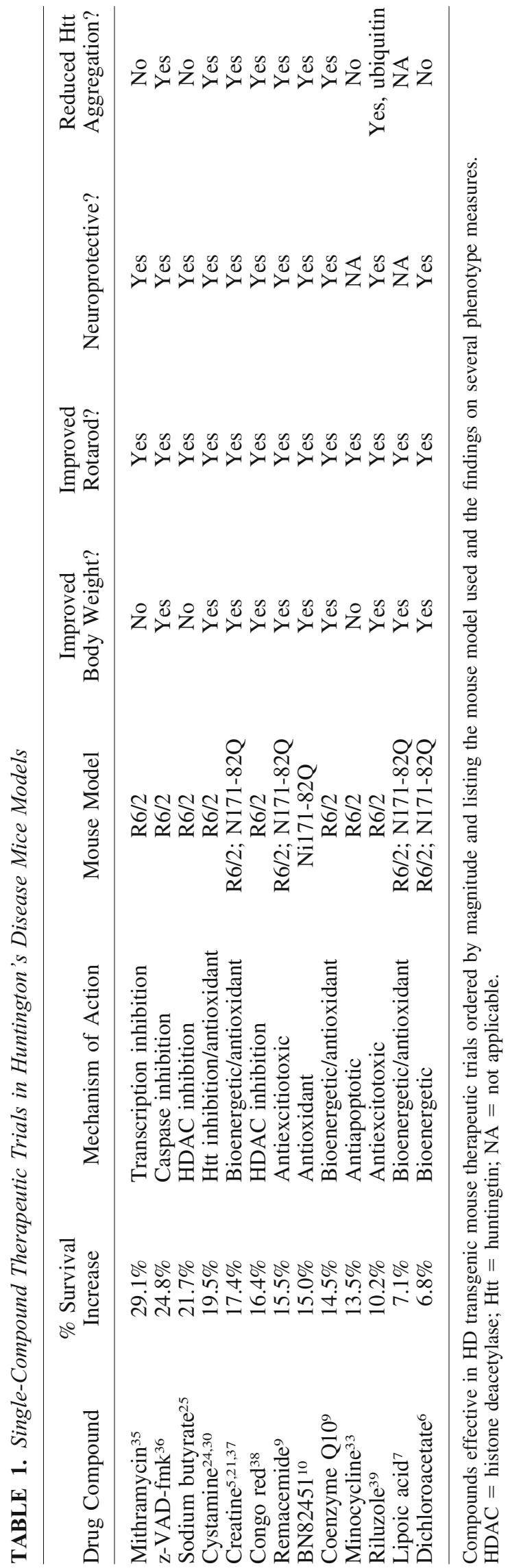

of blood is required to produce sufficient serum for testing levels of agents and it is collected by cardiac puncture, requiring euthanization. HPLC with electrochemical detection along with spectrometric analysis are the methods of choice. ${ }^{21,24}$ Because pharmacokinetic studies require a great deal of effort, our approach has been to use them only when it appears that a compound has real promise for preclinical drug trials proceeding toward human trials. In addition to pharmacokinetic studies, it can be very helpful to perform biochemical studies aimed at determining whether the expected mechanism of action of a compound is actually working. This can be important because many compounds have multiple potential mechanisms of action. It is additionally important to determine whether any of the treatments might work by suppressing the expression of the huntingtin transgene, since this might help the mice but not humans in the absence of identical regulation. This is especially important in mouse lines in which expression is regulated by exogenous promoters and for drugs that affect aggregate formation and gene transcription. ${ }^{25}$ Transgene expression can be analyzed using Western and Northern assays, as well as reverse transcription-polymerase chain reaction methods.

\section{Translation from genetic models to humans}

Many potential therapies have now been tested in genetic models of HD and some have been demonstrated to be neuroprotective in HD transgenic mice (Table 1). Efficacy in mouse trials has provided the rationale for a number of clinical trials that have already occurred or are planned. These include coenzyme Q10, ${ }^{26}$ creatine, remacemide, ${ }^{26}$ riluzole,${ }^{27}$ minocycline, ethyl-eicosapentaenoic acid, ${ }^{28}$ phenylbutyrate, and cysteamine. Most of these have been early-phase clinical trials, so the predictive value of the mouse therapeutic trials are not yet known. However, because these are actually very good models at many levels of analysis, it would be surprising if some compounds benefiting the mouse models did not prove to benefit humans with HD. Since clinical trials may be expensive, effortful, and years in length depending on the type, there is growing discussion within neurodegenerative disorder clinical trials organizations, such as the Huntington Study Group and sponsors about how much preclinical information is enough and how to prioritize among many efficacious compounds. ${ }^{29}$ Of course the bar should be higher for later-phase trials and higher when the interventions have greater potential for risks to human subjects.

The impact therapeutic trials in genetic models can have on selecting compounds for clinical trials in humans depends on many factors relating to the quality and breadth of the preclinical data, as well as on the potential risks and benefits to performing the clinical trials. We provide a schematic to illustrate many of the factors that 


\section{Candidate Compounds}

Selected by mechanistic hypotheses or by screening libraries based on relevant assays

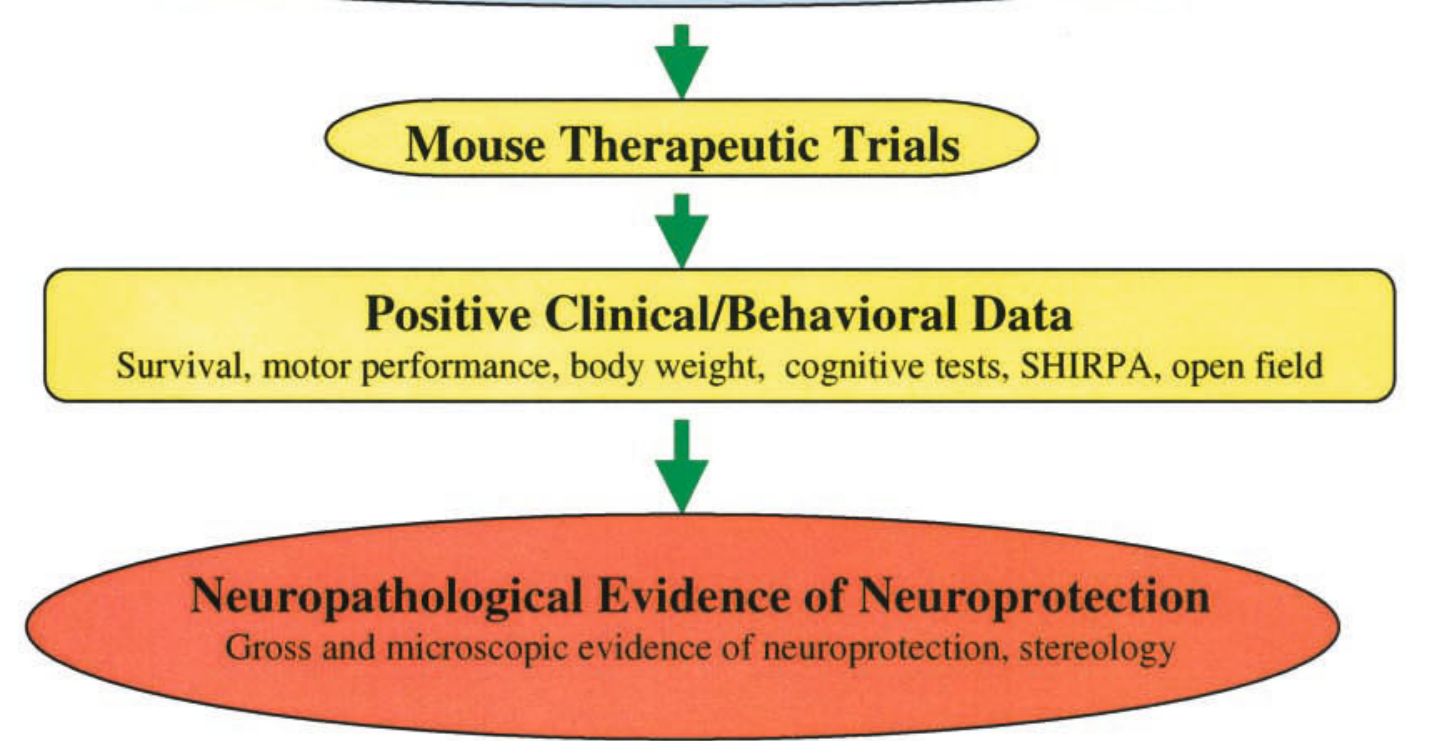

Supplemental Studies

Mechanistic Studies

Pharmacokinetics

Transgene Expression

Biomarker development

\section{Supportive Studies Affecting Priority}

Positive data in submammalian models

Confirmation of primary mouse data by others

Positive data in additional mouse models

Comparative magnitude

Synergy with other candidates

\section{Non-FDA-Approved \\ Preclinical leads \\ Compounds
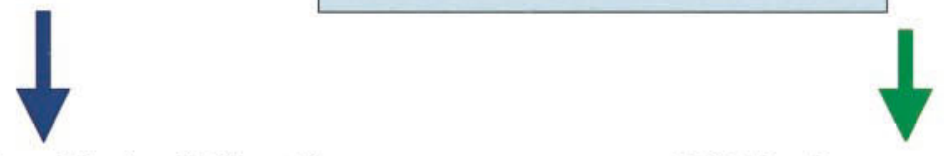

Additional Preclinical Studies

Toxicity

Teratogenicity

Pharmacokinetics

Analog optimization

GMP formulation

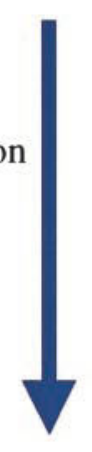

Prioritization
Factors
Availability
Toxicity
Effect magnitude
Redundancy
Mechanism
Synergy

FDA-Approved Compounds

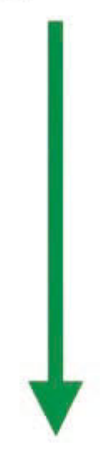

Phase I Clinical Trials

Phase II-III Clinical Trials

FIG. 1. Factors to consider when translating therapies from transgenic mice to humans. 
are important for testing therapies in transgenic mice and considering translation to human clinical trials (FIG. 1). Of course, the highest quality studies and those with the most robust neuroprotection should have the greatest impact. Neuropathological evidence of neuroprotection remains the most important finding with all other outcomes potentially providing some additional support. Since the numbers of compounds demonstrating some efficacy continues to grow, an issue of growing importance is the question of how to compare their efficacy to better prioritize their translation into human studies. Because procedures differ between labs, because the models have many differences, and because even the same mouse line can have different phenotypes in different labs because of genetic drift or background strain differences, it is difficult to compare the magnitude of the effect of one treatment with another. We would propose that laboratories performing therapeutic trials in mouse models use positive controls to help place their results in context. For example, several labs have replicated benefits with cystamine, so including a cystamine treatment cohort in trials of more novel therapeutics could help calibrate results. ${ }^{24,30,31}$

Because the quality of a preclinical therapeutic trial is so dependent on the methodological approaches used, it is vital that publications describing them permit a critical appraisal. This is especially important because results can greatly influence the conduct of human clinical trials. Thus their design, conduct, and analysis must be accurately and fully described. A growing proportion of clinical investigators and journal editors support a published standard for reporting randomized human clinical trials known as the Consolidated Standards of Reporting Clinical Trials (CONSORT) standards. ${ }^{32}$ Although the CONSORT standards include some considerations that have no counterpart in animal studies, they can provide a useful guide to preclinical investigators for reporting their results, and we recommend that investigators familiarize themselves with them. As the numbers of preclinical animal trials increase and conflicting results arise (for example, minocycline studies in HD models ${ }^{33,34}$ ), there may be a need to develop an analogous standard for reporting.

Beyond the results from individual preclinical therapeutic trials, other information that can increase the priority of a compound for translation to humans include replication in additional labs, replication in more than one mouse model, novelty (working through a mechanism for which other drugs are not yet available), evidence of positive synergy with other compounds likely to have human use, positive results in other types of models of disease (toxin models, invertebrates, cell culture), scientific evidence that the mechanism of action might be especially early in the cascades of pathogenesis, low expected toxicity, pharmacokinetic or other information that suggests some comparability in human dosing can be achieved, the potential for a useful biomarker in humans reflective of the biological activity of the compound, and of course constraint-free availability of pharmaceutical-grade compound for human trials.

Acknowledgments: This work was supported by National Institutes of Health Grants NS35255, AT00613, NS045242, NS045806 (to S.M.H. and R.J.F.), AG13846 and AG12992 (to R.J.F.), the Veteran's Administration (R.J.F.), the Huntington's Disease Society of America (S.M.H. and R.J.F.), The Hereditary Disease Foundation (S.M.H. and R.J.F.), The Jerry McDonald Fund in Huntington's Disease (R.J.F.), and a grant from the Mallinckrodt General Clinical Research Center (M01-RR-01066 to S.M.H.).

\section{REFERENCES}

1. Mangiarini L, Sathasivam K, Seller M, Cozens B, Harper A, Hetherington $\mathrm{C}$ et al. Exon 1 of the HD gene with an expanded CAG repeat is sufficient to cause a progressive neurological phenotype in transgenic mice. Cell 87:493-506, 1996.

2. Hockly E, Woodman B, Mahal A, Lewis CM, Bates G. Standardization and statistical approaches to therapeutic trials in the R6/2 mouse. Brain Res Bull 61:469-479, 2003

3. Schilling G, Becher MW, Sharp AH, Jinnah HA, Duan K, Kotzuk $\mathrm{JA}$ et al. Intranuclear inclusions and neuritic aggregates in transgenic mice expressing a mutant $\mathrm{N}$-terminal fragment of huntingtin. Hum Mol Genet 8:397-407, 1999.

4. Yu ZX, Li SH, Evans J, Pillarisetti A, Li H, Li XJ. Mutant huntingtin causes context-dependent neurodegeneration in mice with Huntington's disease. J Neurosci 23:2193-2202, 2003.

5. Andreassen OA, Dedeoglu A, Ferrante RJ, Jenkins BG, Ferrante $\mathrm{KL}$, Thomas $\mathrm{M}$ et al. Creatine increase survival and delays motor symptoms in a transgenic animal model of Huntington's disease. Neurobiol Dis 8:479-491, 2001.

6. Andreassen OA, Ferrante RJ, Huang HM, Dedeoglu A, Park L, Ferrante KL et al. Dichloroacetate exerts therapeutic effects in transgenic mouse models of Huntington's disease. Ann Neurol 50:112-117, 2001.

7. Andreassen OA, Ferrante RJ, Dedeoglu A, Beal MF. Lipoic acid improves survival in transgenic mouse models of Huntington's disease. NeuroReport 12:3371-3373, 2001.

8. Schilling G, Coonfield ML, Ross CA, Borchelt DR. Coenzyme Q10 and remacemide hydrochloride ameliorate motor deficits in a Huntington's disease transgenic mouse model. Neurosci Lett 315: 149-153, 2001

9. Ferrante RJ, Andreassen OA, Dedeoglu A, Ferrante KL, Jenkins BG, Hersch SM et al. Therapeutic effects of coenzyme Q10 and remacemide in transgenic mouse models of Huntington's disease. J Neurosci 22:1592-1599, 2002.

10. Klivenyi P, Ferrante RJ, Gardian G, Browne S, Chabrier PE, Beal MF. Increased survival and neuroprotective effects of BN82451 in a transgenic mouse model of Huntington's disease. J Neurochem 86:267-272, 2003.

11. Duan W, Guo Z, Jiang H, Ware M, Li XJ, Mattson MP. Dietary restriction normalizes glucose metabolism and BDNF levels, slows disease progression, and increases survival in huntingtin mutant mice. Proc Natl Acad Sci USA 100:2911-2916, 2003.

12. Reddy PH, Williams M, Charles V, Garrett L, Pike-Buchanan L, Whetsell WO Jr et al. Behavioural abnormalities and selective neuronal loss in HD transgenic mice expressing mutated fulllength HD cDNA. Nat Genet 20:198-202, 1998.

13. Slow EJ, van Raamsdonk J, Rogers D, Coleman SH, Graham RK, Deng Y et al. Selective striatal neuronal loss in a YAC128 mouse model of Huntington disease. Hum Mol Genet 12:1555-1567, 2003.

14. Li C, Yang W, Gong S, Heintz N. Transgenic mouse models of full-length Huntington's disease gene with BAC modification models. Soc Neurosci Abstr 130.9, 2003. 
15. Lin CH, Tallaksen-Greene S, Chien WM, Cearley JA, Jackson WS, Crouse AB et al. Neurological abnormalities in a knock-in mouse model of Huntington's disease. Hum Mol Genet 10:137144, 2001.

16. Shelbourne PF, Killeen N, Hevner RF, Johnston HM, Tecott L, Lewandoski M et al. A Huntington's disease CAG expansion at the murine Hdh locus is unstable and associated with behavioural abnormalities in mice. Hum Mol Genet 8:763-774, 1999.

17. Wheeler VC, White JK, Gutekunst CA, Vrbanac V, Weaver M, Li $\mathrm{XJ}$ et al. Long glutamine tracts cause nuclear localization of a novel form of huntingtin in medium spiny striatal neurons in HdhQ92 and HdhQ111 knock-in mice. Hum Mol Genet 9:503-513, 2000.

18. Wheeler VC, Gutekunst CA, Vrbanac V, Lebel LA, Schilling G, Hersch $\mathrm{S}$ et al. Early phenotypes that presage late-onset neurodegenerative disease allow testing of modifiers in Hdh CAG knock-in mice. Hum Mol Genet 11:633-640, 2002.

19. Menalled LB, Sison JD, Dragatsis I, Zeitlin S, Chesselet MF. Time course of early motor and neuropathological anomalies in a knock-in mouse model of Huntington's disease with 140 CAG repeats. J Comp Neurol 465:11-26, 2003.

20. Hockly E, Cordery PM, Woodman B, Mahal A, van Dellen A, Blakemore $\mathrm{C}$ et al. Environmental enrichment slows disease progression in R6/2 Huntington's disease mice. Ann Neurol 51:235242, 2002.

21. Dedeoglu A, Kubilus JK, Yang L, Ferrante KL, Hersch SM, Beal MF et al. Creatine therapy provides neuroprotection after onset of clinical symptoms in Huntington's disease transgenic mice. J Neurochem 85:1359-1367, 2003.

22. Rogers DC, Jones DN, Nelson PR, Jones CM, Quilter CA, Robinson TL et al. Use of SHIRPA and discriminant analysis to characterise marked differences in the behavioural phenotype of six inbred mouse strains. Behav Brain Res 105:207-217, 1999.

23. Rogers DC, Peters J, Martin JE, Ball S, Nicholson SJ, Witherden AS et al. SHIRPA, a protocol for behavioral assessment: validation for longitudinal study of neurological dysfunction in mice. Neurosci Lett 306:89-92, 2001.

24. Dedeoglu A, Kubilus JK, Jeitner TM, Matson SA, Bogdanov M, Kowall NW et al. Therapeutic effects of cystamine in a murine model of Huntington's disease. J Neurosci 22:8942-8950, 2002.

25. Ferrante RJ, Kubilus JK, Lee J, Ryu H, Beesen A, Zucker B et al. Histone deacetylase inhibition by sodium butyrate chemotherapy ameliorates the neurodegenerative phenotype in Huntington's disease mice. J Neurosci 23:9418-9427, 2003.

26. Huntington Study Group. A randomized, placebo-controlled trial of coenzyme Q10 and remacemide in Huntington's disease. Neurology 57:397-404, 2001.
27. Konig A. A framework for designing transgenic crops-science, safety and citizen's concerns. Nat Biotechnol 21:1274-1279, 2003.

28. Vaddadi KS, Soosai E, Chiu E, Dingjan P. A randomised, placebocontrolled, double blind study of treatment of Huntington's disease with unsaturated fatty acids. NeuroReport 13:29-33, 2002.

29. Ravina BM, Fagan SC, Hart RG, Hovinga CA, Murphy DD, Dawson TM et al. Neuroprotective agents for clinical trials in Parkinson's disease: a systematic assessment. Neurology 60:12341240, 2003.

30. Karpuj MV, Becher MW, Springer JE, Chabas D, Youssef S, Pedotti R et al. Prolonged survival and decreased abnormal movements in transgenic model of Huntington disease, with administration of the transglutaminase inhibitor cystamine. Nat Med 8:143-149, 2002.

31. Mastroberardino PG, Iannicola C, Nardacci R, Bernassola F, De Laurenzi V, Melino $\mathrm{G}$ et al. 'Tissue' transglutaminase ablation reduces neuronal death and prolongs survival in a mouse model of Huntington's disease. Cell Death Differ 9:873-880, 2002.

32. Altman D, Schulz K, Mosher D, Eggar M, Davidoff F, Elbourne D et al. The revised CONSORT statement for reporting randomized trials: explanations and elaboration. Ann Intern Med 134:663-694, 2001.

33. Chen M, Ona VO, Li M, Ferrante RJ, Fink KB, Zhu S et al. Minocycline inhibits caspase- 1 and caspase- 3 expression and delays mortality in a transgenic mouse model of Huntington disease. Nat Med 6:797-801, 2000

34. Smith DL, Woodman B, Mahal A, Sathasivam K, Ghazi-Noori S, Lowden PA et al. Minocycline and doxycycline are not beneficial in a model of Huntington's disease. Ann Neurol 54:186-196, 2003.

35. Ferrante RJ, Kubilus JK, Kugars KL, Rubinsztein DC, Ryu H, Lee $\mathrm{JH}$ et al. Therapeutic effects of mithramycin in R6/2 transgenic Huntington's disease mice. Soc Neurosci Abstr 28, 2002.

36. Ona VO, Li M, Vonsattel JP, Andrews LJ, Khan SQ, Chung WM et al. Inhibition of caspase-1 slows disease progression in a mouse model of Huntington's disease. Nature 399:263-267, 1999.

37. Ferrante RJ, Andreassen OA, Jenkins BG, Dedeoglu A, Kuemmerle S, Kubilus JK et al. Neuroprotective effects of creatine in a transgenic mouse model of Huntington's disease. J Neurosci 20: 4389-4397, 2000.

38. Sanchez I, Mahlke C, Yuan J. Pivotal role of oligomerization in expanded polyglutamine neurodegenerative disorders. Nature 421: 373-379, 2003

39. Schiefer J, Landwehrmeyer GB, Luesse HG, Sprunken A, Puls C, Milkereit A et al. Riluzole prolongs survival time and alters nuclear inclusion formation in a transgenic mouse model of Huntington's disease. Mov Disord 17:748-757, 2002. 